\title{
Hermitean Téodorescu Transform Decomposition of Continuous Matrix Functions on Fractal Hypersurfaces
}

\author{
Ricardo Abreu-Blaya, ${ }^{1}$ Juan Bory-Reyes, ${ }^{2}$ Fred Brackx, ${ }^{3}$ \\ and Hennie De Schepper ${ }^{3}$ \\ ${ }^{1}$ Facultad de Informática y Matemática, Universidad de Holguín, Holguín 80100, Cuba \\ ${ }^{2}$ Departamento de Matemática, Universidad de Oriente, Santiago de Cuba 90500, Cuba \\ ${ }^{3}$ Clifford Research Group, Department of Mathematical Analysis, Faculty of Engineering, \\ Ghent University, Galglaan 2, 9000 Gent, Belgium
}

Correspondence should be addressed to Hennie De Schepper, hennie.deschepper@ugent.be

Received 18 December 2009; Accepted 12 April 2010

Academic Editor: Michel C. Chipot

Copyright (C) 2010 Ricardo Abreu-Blaya et al. This is an open access article distributed under the Creative Commons Attribution License, which permits unrestricted use, distribution, and reproduction in any medium, provided the original work is properly cited.

We consider Hölder continuous circulant $(2 \times 2)$ matrix functions $\mathbf{G}_{2}^{1}$ defined on the fractal boundary $\Gamma$ of a domain $\Omega$ in $\mathbb{R}^{2 n}$. The main goal is to study under which conditions such a function $G_{2}^{1}$ can be decomposed as $G_{2}^{1}=G_{2}^{1+}-G_{2}^{1-}$, where the components $G_{2}^{1 \pm}$ are extendable to $\mathrm{H}$-monogenic functions in the interior and the exterior of $\Omega$, respectively. H-monogenicity are a concept from the framework of Hermitean Clifford analysis, a higher-dimensional function theory centered around the simultaneous null solutions of two first-order vector-valued differential operators, called Hermitean Dirac operators. H-monogenic functions then are the null solutions of a $(2 \times 2)$ matrix Dirac operator, having these Hermitean Dirac operators as its entries; such matrix functions play an important role in the function theoretic development of Hermitean Clifford analysis. In the present paper a matricial Hermitean Téodorescu transform is the key to solve the problem under consideration. The obtained results are then shown to include the ones where domains with an Ahlfors-David regular boundary were considered.

\section{Introduction}

Clifford analysis is a higher-dimensional function theory offering a generalization of the theory of holomorphic functions in the complex plane and, at the same time, a refinement of classical harmonic analysis. The standard case, also referred to as Euclidean Clifford analysis, focuses on the null solutions, called monogenic functions, of the vector-valued Dirac operator $\partial_{\underline{X}}=\sum_{j=1}^{m} e_{j} \partial_{x_{j}}$, which factorizes the $m$-dimensional Laplacian: $\partial_{\underline{X}}^{2}=-\Delta_{m}$. Here 
$\left(e_{1}, \ldots, e_{m}\right)$ is an orthonormal basis for the quadratic space $\mathbb{R}^{0, m}$ underlying the construction of the real Clifford algebra $\mathbb{R}_{0, m}$, where the considered functions take their values. Since the Dirac operator is invariant with respect to the action of the orthogonal group $\mathrm{O}(m ; \mathbb{R})$, doubly covered by the Pin $(m)$ group of the Clifford algebra $\mathbb{R}_{0, m}$, the resulting function theory is said to be rotation invariant. Standard references for Euclidean Clifford analysis are [1-5].

More recently, Hermitean Clifford analysis has emerged as yet a refinement of the Euclidean case. One of the ways for introducing it is by considering the complex Clifford algebra $\mathbb{C}_{2 n}$, equipped with a complex structure, that is, an $\mathrm{SO}(2 n ; \mathbb{R})$ element $J$ for which $J^{2}=-1$. In fact, it is precisely in order to ensure that such a complex structure exists that the dimension of the underlying vector space is taken to be even. The resulting function theory focuses on the simultaneous null solutions of two complex Hermitean Dirac operators $\partial_{Z}$ and $\partial_{Z^{\dagger}}$ which no longer factorize but still decompose the Laplace operator in the sense that $4\left(\partial_{\underline{Z}} \partial_{Z^{\dagger}}+\partial_{Z^{\dagger}} \partial_{Z}\right)=\Delta_{2 n}$. Since the system indeed constitutes a refinement of the original Euclidean Dirac equation, the fundamental group invariance of this system breaks down to a smaller group; it was shown in [6] that it concerns the unitary group $\mathrm{U}(n ; \mathbb{C})$. The study of complex Dirac operators was initiated in [7-10]; a systematic development of the associated function theory still is in full progress; see, for example, [6, 11-15].

In [16] a Cauchy integral formula for Hermitean monogenic functions was established, obviously an essential result in the development of the function theory. However, as in some very particular cases Hermitean monogenicity is equivalent with (anti)holomorphy in $n$ complex variables $\left(z_{1}, \ldots, z_{n}\right)$ (see [12]), such a representation formula could not take the traditional form as in the complex plane or in Euclidean Clifford analysis. The matrix approach needed to obtain the desired result leads to the concept of (left or right) $\mathbf{H}$ monogenic functions, introduced as circulant $(2 \times 2)$ matrix functions, which are (left or right) null solutions of a $(2 \times 2)$ circulant matrix Dirac operator, having the Hermitean Dirac operators $\partial_{\underline{Z}}$ and $\partial_{Z^{\dagger}}$ as its entries. Although the $\mathbf{H}$-monogenic system thus arose as an auxiliary concept in Hermitean Clifford analysis, it was meanwhile also further studied itself; see also $[15,17,18]$.

In the present paper, we consider Hölder continuous circulant $(2 \times 2)$ matrix functions $\mathrm{G}_{2}^{1}$ defined on the fractal boundary $\Gamma$ of a domain $\Omega$ in $\mathbb{R}^{2 n}$, and we investigate under

which conditions such a function $G_{2}^{1}$ can be decomposed as $G_{2}^{1}=G_{2}^{1^{+}}-G_{2}^{1^{-}}$, where the components $\mathbf{G}_{2}^{1^{ \pm}}$are extendable to $\mathbf{H}$-monogenic functions in the interior and the exterior of $\Omega$, respectively. This type of decomposition (or "jump") problem has already been considered in Euclidean Clifford analysis in, for example, [19-22] for domains with boundaries showing minimal smoothness, including some results for fractal boundaries as well. In [23] a similar decomposition problem for domains with fractal boundaries was considered in the Hermitean Clifford context, the approach, however, not being suited for a treatment of the circulant matrix situation. It turns out that the introduction of a matricial Hermitean Téodorescu transform is crucial to solve this problem.

\section{Preliminaries}

\subsection{Some Elements of Hermitean Clifford Analysis}

Let $\left(e_{1}, \ldots, e_{m}\right)$ be an orthonormal basis of Euclidean space $\mathbb{R}^{m}$ and consider the complex Clifford algebra $\mathbb{C}_{m}$ constructed over $\mathbb{R}^{m}$. The noncommutative or geometric multiplication 
in $\mathbb{C}_{m}$ is governed by the following rules:

$$
\begin{aligned}
& e_{j}^{2}=-1, \quad j=1, \ldots, m, \\
& e_{j} e_{k}+e_{k} e_{j}=0, \quad j \neq k .
\end{aligned}
$$

The Clifford algebra $\mathbb{C}_{m}$ thus is generated additively by elements of the form $e_{A}=e_{j_{1}} \cdots e_{j_{k}}$, where $A=\left\{j_{1}, \ldots, j_{k}\right\} \subset\{1, \ldots, m\}$ is such that $j_{1}<\cdots<j_{k}$, while for $A=\emptyset$, one puts $e_{\emptyset}=1$, the identity element. The dimension of $\mathbb{C}_{m}$ thus is $2^{m}$. Any Clifford number $\lambda \in \mathbb{C}_{m}$ may thus be written as $\lambda=\sum_{A} \lambda_{A} e_{A}, \lambda_{A} \in \mathbb{C}$, and its Hermitean conjugate $\lambda^{\dagger}$ is defined by

$$
\lambda^{\dagger}=\sum_{A} \lambda_{A}^{c} \bar{e}_{A}
$$

where the bar denotes the real Clifford algebra conjugation, that is, the main anti-involution for which $\bar{e}_{j}=-e_{j}$, and $\lambda_{A}^{c}$ stands for the complex conjugate of the complex number $\lambda_{A}$.

Euclidean space $\mathbb{R}^{m}$ is embedded in the Clifford algebra $\mathbb{C}_{m}$ by identifying $\left(x_{1}, \ldots, x_{m}\right)$ with the real Clifford vector $\underline{X}$ given by $\underline{X}=\sum_{j=1}^{m} e_{j} x_{j}$, for which $\underline{X}^{2}=-\langle\underline{X}, \underline{X}\rangle=-|\underline{X}|^{2}$. The Fischer dual of the vector $\underline{X}$ is the vector-valued first-order Dirac operator $\partial_{\underline{X}}=\sum_{j=1}^{m} e_{j} \partial_{x_{j}}$, factorizing the Laplacian: $\Delta_{m}=-\partial_{X}^{2}$; it is precisely this Dirac operator which underlies the notion of monogenicity of a function, the higher-dimensional counterpart of holomorphy in the complex plane. The functions under consideration are defined on (open subsets of) $\mathbb{R}^{m}$ and take values in the Clifford algebra $\mathbb{C}_{m}$. They are of the form $g=\sum_{A} g_{A} e_{A}$, where the functions $g_{A}$ are complex valued. Whenever a property such as continuity and differentiability is ascribed to $g$, it is meant that all the components $g_{A}$ possess the cited property. A Clifford algebra-valued function $g$, defined and differentiable in an open region $\Omega$ of $\mathbb{R}^{m}$, is then called (left) monogenic in $\Omega$ if and only if $\partial_{\underline{X}} g=0$ in $\Omega$.

The transition from Euclidean Clifford analysis as described above to the Hermitean Clifford setting is essentially based on the introduction of a complex structure $J$. This is a particular $\mathrm{SO}(m)$ element, satisfying $J^{2}=-\mathbf{1}_{m}$. Since such an element cannot exist when the dimension $m$ of the vector space is odd, we will put $m=2 n$ from now on. In terms of the chosen orthonormal basis, a particular realization of the complex structure may be $J\left[e_{2 j-1}\right]=-e_{2 j}$ and $J\left[e_{2 j}\right]=e_{2 j-1}, j=1, \ldots, n$. Two projection operators $\pm(1 / 2)\left(\mathbf{1}_{2 n} \pm i J\right)$ associated to this complex structure $J$ then produce the main objects of Hermitean Clifford analysis by acting upon the corresponding objects in the Euclidean setting; see [11,12]. First of all, the vector space $\mathbb{C}^{2 n}$ thus decomposes as $W^{+} \oplus W^{-}$into two isotropic subspaces. The real Clifford vector $\underline{X}$ is now denoted by

$$
\underline{X}=\sum_{j=1}^{n}\left(e_{2 j-1} x_{2 j-1}+e_{2 j} x_{2 j}\right)
$$

and its corresponding Dirac operator $\partial_{\underline{X}}$ by

$$
\partial_{\underline{X}}=\sum_{j=1}^{n}\left(e_{2 j-1} \partial_{x_{2 j-1}}+e_{2 j} \partial_{x_{2 j}}\right)
$$


while we will also consider their so-called "twisted" counterparts, obtained through the action of $J$, that is,

$$
\begin{gathered}
\underline{X} \mid=\sum_{j=1}^{n}\left(e_{2 j-1} x_{2 j}-e_{2 j} x_{2 j-1}\right), \\
\partial_{\underline{X} \mid}=\sum_{j=1}^{n}\left(e_{2 j-1} \partial_{x_{2 j}}-e_{2 j} \partial_{x_{2 j-1}}\right) .
\end{gathered}
$$

As was the case with $\partial_{\underline{X}}$, a notion of monogenicity may be associated in a natural way to $\partial_{\underline{X}}$ as well. The projections of the vector variable $\underline{X}$ and the Dirac operator $\partial_{\underline{X}}$ on the spaces $W^{ \pm}$ then give rise to the Hermitean Clifford variables $\underline{Z}$ and $\underline{Z}^{\dagger}$, given by

$$
\underline{Z}=\frac{1}{2}(\underline{X}+i \underline{X} \mid), \quad \underline{Z}^{\dagger}=-\frac{1}{2}(\underline{X}-i \underline{X} \mid),
$$

and (up to a factor) to the Hermitean Dirac operators $\partial_{\underline{Z}}$ and $\partial_{\underline{Z^{\dagger}}}$ given by

$$
\partial_{\underline{Z}^{\dagger}}=\frac{1}{4}\left(\partial_{\underline{X}}+i \partial_{\underline{X} \mid}\right), \quad \partial_{\underline{Z}}=-\frac{1}{4}\left(\partial_{\underline{X}}-i \partial_{\underline{X} \mid}\right)
$$

(see $[6,11])$. Observe for further use that the Hermitean vector variables and Dirac operators are isotropic, that is, $(\underline{Z})^{2}=\left(\underline{Z}^{\dagger}\right)^{2}=0$ and $\left(\partial_{\underline{Z}}\right)^{2}=\left(\partial_{\underline{Z}^{\dagger}}\right)^{2}=0$, whence the Laplacian allows for the decomposition $\Delta_{2 n}=4\left(\partial_{\underline{Z}} \partial_{\underline{Z}^{\dagger}}+\partial_{\underline{Z}^{\dagger}} \partial_{\underline{Z}}\right)$, while also $\underline{Z} \underline{Z}^{\dagger}+\underline{Z}^{\dagger} \underline{Z}=|\underline{Z}|^{2}=\left|\underline{Z}^{\dagger}\right|^{2}=|\underline{X}|^{2}$. These objects lie at the core of the Hermitean function theory by means of the following definition (see, e.g., $[6,11])$.

Definition 2.1. A continuously differentiable function $g$ in $\Omega \subset \mathbb{R}^{2 n}$ with values in $\mathbb{C}_{2 n}$ is called left Hermitean monogenic (or left $h$-monogenic) in $\Omega$, if and only if it satisfies in $\Omega$ the system

$$
\partial_{\underline{Z}} g=0=\partial_{\underline{Z}^{\dagger}} g
$$

or, equivalently, the system

$$
\partial_{\underline{X}} g=0=\partial_{\underline{X}} g \text {. }
$$

In a similar way right $h$-monogenicity is defined. Functions which are both left and right $h$-monogenic are called two-sided $h$-monogenic. This definition inspires the statement that $h$ monogenicity constitutes a refinement of monogenicity, since $h$-monogenic functions (either left or right) are monogenic w.r.t. both Dirac operators $\partial_{\underline{X}}$ and $\partial_{\underline{X}}$.

In what follows, we will systematically take $\Omega \subset \overline{\mathbb{R}}^{2 n}$ to be a so-called Jordan domain, that is, a bounded oriented connected open subset of $\mathbb{R}^{2 n}$, the boundary $\Gamma$ of which is a compact topological surface. Note that, in the case $n=1$, this notion coincides with the usual one of a Jordan domain in the complex plane. For further use, we also introduce the notation $\Omega^{+} \equiv \Omega$, and $\Omega^{-} \equiv \mathbb{R}^{2 n} \backslash \bar{\Omega}$. 


\subsection{Some Elements of the Matricial Hermitean Clifford Setting}

The fundamental solutions of the Dirac operators $\partial_{\underline{X}}$ and $\partial_{\underline{X} \mid}$ are, respectively, given by

$$
E(\underline{X})=-\frac{1}{\sigma_{2 n}} \frac{\underline{X}}{|\underline{X}|^{2 n}}, \quad E \mid(\underline{X})=-\frac{1}{\sigma_{2 n}} \frac{\underline{X} \mid}{|\underline{X}|^{2 n}}, \quad \underline{X} \in \mathbb{R}^{2 n} \backslash\{0\},
$$

where $\sigma_{2 n}$ denotes the surface area of the unit sphere in $\mathbb{R}^{2 n}$. Introducing the functions $\varepsilon=$ $-(E+i E \mid)$ and $\mathcal{E}^{\dagger}=(E-i E \mid)$, explicitly given by

$$
\mathcal{E}(\underline{Z})=\frac{2}{\sigma_{2 n}} \frac{\underline{Z}}{|\underline{Z}|^{2 n}}, \quad \mathcal{E}^{\dagger}(\underline{Z})=\frac{2}{\sigma_{2 n}} \frac{\underline{Z}^{\dagger}}{|\underline{Z}|^{2 n}}
$$

it is directly seen that $\mathcal{\varepsilon}$ and $\mathcal{E}^{\dagger}$ are not the fundamental solutions to the respective Hermitean Dirac operators $\partial_{\underline{Z}}$ and $\partial_{Z^{\dagger}}$. Surprisingly, however, introducing the particular circulant $(2 \times 2)$ matrices

$$
\boldsymbol{\Phi}_{\left(\underline{Z}, \underline{Z}^{\dagger}\right)}=\left(\begin{array}{cc}
\partial_{\underline{Z}} & \partial_{\underline{Z}^{\dagger}} \\
\partial_{\underline{Z}^{\dagger}} & \partial_{\underline{Z}}
\end{array}\right), \quad \boldsymbol{\varepsilon}=\left(\begin{array}{cc}
\varepsilon & \varepsilon^{\dagger} \\
\mathcal{E}^{\dagger} & \varepsilon
\end{array}\right), \quad \delta=\left(\begin{array}{ll}
\delta & 0 \\
0 & \delta
\end{array}\right),
$$

where $\delta$ is the Dirac delta distribution, one obtains that $\boldsymbol{\Phi}_{\left(\underline{Z}, Z^{\dagger}\right)} \boldsymbol{\varepsilon}(\underline{Z})=\boldsymbol{\delta}(\underline{Z})$, so that $\mathcal{\varepsilon}$ may be considered as a fundamental solution of $\boldsymbol{\vartheta}_{\left(\underline{Z}, \underline{Z}^{\dagger}\right)}$ in a matricial context, see, for example, $[8,16,18]$. Moreover, the Dirac matrix $\boldsymbol{\Phi}_{\left(\underline{Z}, \underline{Z}^{\dagger}\right)}$ in some sense factorizes the Laplacian, since

$$
4 \boldsymbol{\Phi}_{\left(\underline{Z}, \underline{Z}^{\dagger}\right)}\left(\boldsymbol{\Phi}_{\left(\underline{Z}, \underline{Z}^{\dagger}\right)}\right)^{\dagger}=\left(\begin{array}{cc}
\Delta_{2 n} & 0 \\
0 & \Delta_{2 n}
\end{array}\right) \equiv \boldsymbol{\Delta}
$$

where $\Delta_{2 n}$ is the usual Laplace operator in $\mathbb{R}^{2 n}$. It was exactly this simple observation which leads to the idea of following a matrix approach in order to establish integral representation formulae in the Hermitean setting; see $[15,16]$. Moreover, it inspired the following definition.

Definition 2.2. Let $g_{1}, g_{2}$ be continuously differentiable functions defined in $\Omega$ and taking values in $\mathbb{C}_{2 n}$, and consider the matrix function:

$$
\mathbf{G}_{2}^{1}=\left(\begin{array}{ll}
g_{1} & g_{2} \\
g_{2} & g_{1}
\end{array}\right)
$$

Then $\mathrm{G}_{2}^{1}$ is called left (resp., right) $\mathbf{H}$-monogenic in $\Omega$ if and only if it satisfies in $\Omega$ the system

$$
\boldsymbol{\Phi}_{\left(\underline{Z}, \underline{Z}^{\dagger}\right)} \mathbf{G}_{2}^{1}=\mathbf{O} \quad\left(\text { resp., } \mathbf{G}_{2}^{1} \boldsymbol{\Phi}_{\left(\underline{Z}, \underline{Z}^{\dagger}\right)}=\mathbf{O}\right)
$$

Here $\mathbf{O}$ denotes the matrix with zero entries. 
Explicitly, the system for left $\mathbf{H}$-monogenicity reads:

$$
\begin{aligned}
& \partial_{\underline{Z}}\left[g_{1}\right]+\partial_{\underline{Z}^{\dagger}}\left[g_{2}\right]=0, \\
& \partial_{\underline{Z}^{\dagger}}\left[g_{1}\right]+\partial_{\underline{Z}}\left[g_{2}\right]=0 .
\end{aligned}
$$

Again, a notion of two-sided $\mathbf{H}$-monogenicity may be defined similarly. However, unless mentioned explicitly, we will only work with left $\mathbf{H}$-monogenic matrix functions. This matrix approach has also been successfully applied in $[17,24]$ for the construction of a boundary values theory of $h$-monogenic functions.

Observe however that the $\mathbf{H}$-monogenicity of the matrix function $\mathbf{G}_{2}^{1}$ does not imply the $h$-monogenicity of its entry functions $g_{1}$ and $g_{2}$. Nevertheless, choosing in particular $g_{1}=$ $g$ and $g_{2}=0$, the $\mathbf{H}$-monogenicity of the corresponding diagonal matrix, denoted by $\mathbf{G}_{0}$, is seen to be equivalent to the $h$-monogenicity of the function $g$. Moreover, considering the matricial Laplacian introduced in (2.13), one may call a matrix function $\mathrm{G}_{2}^{1}$ harmonic if and only if it satisfies the equation $\Delta\left[G_{2}^{1}\right]=\mathbf{O}$. Each $H$-monogenic matrix function $G_{2}^{1}$ then is harmonic, ensuring that its entries are harmonic functions in the usual sense.

In general, notions of continuity, differentiability, and integrability of $G_{2}^{1}$ are introduced by means of the corresponding notions for its entries. In what follows, we will in particular use the notations $\mathcal{C}^{0, v}(\Gamma), \mathcal{C}^{0, v}\left(\mathbb{R}^{2 n}\right)$, and $\mathbf{L}_{p}(\Omega)$ for the class of Hölder continuous and $p$-integrable circulant matrix functions, respectively.

\subsection{Some Elements of Fractal Geometry}

Let $\mathbf{E}$ be an arbitrary subset of $\mathbb{R}^{2 n}$. Then for any $s \geq 0$ its Hausdorff measure $\mathscr{L}^{s}(\mathbf{E})$ may be defined by

$$
\mathscr{H}^{S}(\mathbf{E})=\lim _{\delta \rightarrow \mathbf{0}} \inf \left\{\sum_{\mathbf{k}=\mathbf{1}}^{\infty}\left(\operatorname{diam} \mathbf{B}_{\mathbf{k}}\right)^{\mathrm{s}}: \mathbf{E} \subset \bigcup_{\mathbf{k}=\mathbf{1}}^{\infty} \mathbf{B}_{\mathbf{k}}, \operatorname{diam} \mathbf{B}_{\mathbf{k}}<\delta\right\},
$$

where the infimum is taken over all countable $\delta$-coverings $\left\{B_{k}\right\}$ of $\mathbf{E}$ with open or closed balls. Note that, for $s=2 n$, the Hausdorff measure $\mathfrak{L}^{2 n}$ coincides, up to a positive multiplicative constant, with the Lebesgue measure $\mathcal{L}^{2 n}$ in $\mathbb{R}^{2 n}$.

Now, let $\mathbf{E}$ be a compact subset of $\mathbb{R}^{2 n}$. The Hausdorff dimension of $\mathbf{E}$, denoted by $\alpha_{H}(\mathbf{E})$, is then defined as the infimum of all $s \geq 0$ such that $\mathfrak{L}^{s}(\mathbf{E})<\infty$. For more details concerning the Hausdorff measure and dimension we refer the reader to [25, 26].

Frequently, however, see, for example, [27], the so-called box dimension is more appropriated than the Hausdorff dimension to measure the roughness of a given set $E$. By definition, the box dimension of a compact set $\mathbf{E} \subset \mathbb{R}^{2 n}$ is equal to

$$
\alpha(\mathbf{E})=\lim _{\varepsilon \rightarrow 0} \sup \frac{\log \mathbf{N}_{\mathbf{E}}(\varepsilon)}{-\log \varepsilon}
$$


where $\mathbf{N}_{\mathrm{E}}(\varepsilon)$ stands for the minimal number of $\varepsilon$-balls needed to cover $\mathbf{E}$. Note that the limit in (2.18) remains unchanged if $\mathbf{N}_{\mathrm{E}}(\varepsilon)$ is replaced by the number of $k$-cubes, with $2^{-k} \leq \varepsilon<2^{-k+1}$, intersecting E. For completeness we recall that a cube $Q$ is called a $k$-cube if it is of the form

$$
\left[l_{1} 2^{-k},\left(l_{1}+1\right) 2^{-k}\right] \times \cdots \times\left[l_{2 n} 2^{-k},\left(l_{2 n}+1\right) 2^{-k}\right]
$$

where $k$ and $l_{1}, \ldots, l_{2 n}$ are integers. The box dimension and the Hausdorff dimension of a given compact set $\mathbf{E}$ can be equal, which is, for instance, the case for the so-called $(2 n-1)$ rectifiable sets (see [28]), but this is not the case in general, where we have that $\alpha_{H}(\mathbf{E}) \leq \alpha(\mathbf{E})$.

In what follows we will assume the boundary $\Gamma$ of our Jordan domain $\Omega$ to have Hausdorff dimension $2 n-1 \leq \alpha_{H}(\Gamma)<2 n$. This includes the case when $\Gamma$ is fractal in the sense of Mandelbrot, that is, when $2 n-1<\alpha_{H}(\Gamma)$.

\section{A Pair of Euclidean Téodorescu Transforms}

From now on we reserve the notations $\underline{Y}$ and $\underline{Y} \mid$ for Clifford vectors associated to points in $\Omega^{ \pm}$. We may then consider the Euclidean Téodorescu transforms $\tau_{\Omega} g$ and $\tau_{\Omega} \mid g$ of a function $g$, assumed to be integrable in $\Omega$, given by

$$
\begin{gathered}
\tau_{\Omega} g(\underline{X})=-\int_{\Omega} E(\underline{Y}-\underline{X}) g(\underline{Y}) d V(\underline{Y}), \\
\tau_{\Omega}\left|g(\underline{X})=-\int_{\Omega} E\right|(\underline{Y}-\underline{X}) g(\underline{Y}) d V(\underline{Y} \mid),
\end{gathered}
$$

where $d V(\underline{Y})$ and $d V(\underline{Y} \mid)$ are oriented volume elements on $\Omega$, for which it is easily checked that $d V(\underline{Y})=d V(\underline{Y} \mid)$. For the sake of completeness, we recall some basic properties of $\tau_{\Omega}$ and $\tau_{\Omega}$, which are generalizations to the case of Clifford analysis of the well-known properties established in the complex plane.

To this end, let $g$ be a $\mathbb{C}_{2 n}$-valued function defined on $\Gamma$, which satisfies a Hölder condition of order $v$, that is, $g \in C^{0, v}(\Gamma)$, where $0<v \leq 1$, and denote by $\tilde{g}$ the so-called Whitney extension of $g$ from $\Gamma$ to the whole of $\mathbb{R}^{2 n}$ (see [29]). We recall that the Whitney extension of $g \in C^{0, v}(\Gamma)$ is a compactly supported function $\tilde{g} \in C^{\infty}\left(\mathbb{R}^{2 n} \backslash \Gamma\right) \cap C^{0, v}\left(\mathbb{R}^{2 n}\right)$ for which it holds that $\left.\tilde{g}\right|_{\Gamma}=g$ and

$$
\left|\partial_{x_{i}} \tilde{g}(\underline{X})\right| \leq c \operatorname{dist}(\underline{X}, \Gamma)^{\nu-1}, \quad \text { for } \underline{X} \in \mathbb{R}^{2 n} \backslash \Gamma, i=1, \ldots, 2 n \text {. }
$$

We then first formulate an auxiliary result.

Lemma 3.1. $\partial_{\underline{X}} \tilde{g}$ and $\partial_{\underline{X}} \mid \tilde{g}$ are $p$-integrable in $\Omega$ for any $p<(2 n-\alpha(\Gamma)) /(1-v)$.

Proof. We only give the main lines of the proof; for details we refer the reader to [22, Lemma 6.1]. In the notation of [30], let $\mathcal{W}=\bigcup_{k} \mathcal{W}^{k}$ be the Whitney partition of $\Omega$ by means of $k$-cubes. We then have

$$
\int_{\Omega}\left|\partial_{\underline{Y}} \underline{\tilde{g}}\right|^{p} d V(\underline{Y})=\sum_{Q \in \mathcal{W}} \int_{Q}\left|\partial_{\underline{Y}} \tilde{g}\right|^{p} d V(\underline{Y}) .
$$


On the other hand, (3.2) implies that for $Q \in \mathcal{W}^{k}$

$$
\int_{Q}\left|\partial_{\underline{Y}} \tilde{g}\right|^{p} d V(\underline{Y}) \leq c \int_{Q} \operatorname{dist}(\underline{Y}, \Gamma)^{p(v-1)} d V(\underline{Y}) \leq c 2^{k(p(1-v)-2 n)},
$$

since $\operatorname{dist}(\underline{Y}, \Gamma) \simeq 2^{-k}$. Now, invoking the fact that the number of $k$-cubes appearing in $\mathcal{W}^{k}$ is less than $c N_{\Gamma}\left(2^{-k}\right)$, while by definition of $\alpha(\Gamma)$,

$$
N_{\Gamma}\left(2^{-k}\right) \leq c 2^{k \alpha^{\prime}}
$$

for any $\left.\alpha^{\prime} \in\right] \alpha(\Gamma), 2 n[$, we arrive at

$$
\int_{\Omega}\left|\partial_{\underline{Y}} \tilde{g}\right|^{p} d V(\underline{Y}) \leq c \sum_{k=0}^{\infty} N_{\Gamma}\left(2^{-k}\right) 2^{k(p(1-v)-2 n)} \leq c \sum_{k=0}^{\infty} 2^{k\left(p(1-v)-2 n+\alpha^{\prime}\right)}
$$

the last series being convergent for $p<\left(2 n-\alpha^{\prime}\right) /(1-v)$. In view of the arbitrary choice of $\alpha^{\prime}$, this concludes the proof.

Now, take $v$ such that

$$
v>\frac{\alpha(\Gamma)}{2 n}
$$

and then it holds that $(2 n-\alpha(\Gamma)) /(1-v)>2 n$; whence, on account of the previous lemma, there exist exponents $p>2 n$ such that $\partial_{\underline{X}} \tilde{g}$ and $\partial_{\underline{X}} \tilde{g}$ are $p$-integrable in $\Omega$. From this observation it then follows that, for $g \in C^{0, v}(\Gamma)$, with $v$ as in (3.7), both $\tau_{\Omega} \partial_{\underline{X}} \tilde{g}$ and $\tau_{\Omega}\left|\partial_{\underline{X}}\right| \tilde{g}$ belong to $C^{0, \mu}\left(\mathbb{R}^{2 n}\right)$, for any $\mu$ satisfying

$$
\mu<\frac{2 n v-\alpha(\Gamma)}{2 n-\alpha(\Gamma)}
$$

due to the fact that the Téodorescu transform maps the space of $p$-integrable functions with compact support to $C^{0,(p-2 n) / p}\left(\mathbb{R}^{2 n}\right)$ if $p>2 n$ (see, e.g., [5]). The following result then holds.

Proposition 3.2. For $g \in C^{0, v}(\Gamma)$, with $v$ as in (3.7), consider

$$
\begin{gathered}
\Phi(\underline{X})=\chi(\underline{X}) \tilde{g}(\underline{X})-\tau_{\Omega} \partial_{\underline{X}} \tilde{g}(\underline{X}), \\
\Phi\left|(\underline{X})=\chi(\underline{X}) \tilde{g}(\underline{X})-\tau_{\Omega}\right| \partial_{\underline{X}} \mid \tilde{g}(\underline{X}),
\end{gathered}
$$

With $X(\underline{X})$ denoting the characteristic function of the set $\Omega$. Then $\Phi(\underline{X})$ and $\Phi \mid(\underline{X})$ are monogenic in $\Omega^{+}$and in $\Omega^{-}$, with respect to $\partial_{\underline{X}}$ and $\partial_{\underline{X}}$, respectively. They are continuous in the corresponding closed domains, vanish at infinity, and show jump g over the boundary $\Gamma$.

Proof. For simplicity we restrict ourselves to $\Phi(\underline{X})$, the proof for $\Phi \mid(\underline{X})$ running along similar lines. The continuity of $\Phi$ on the closed domains follows from the fact that $\tau_{\Omega} \partial_{\underline{X}} \tilde{g} \in C^{0, \mu}\left(\mathbb{R}^{2 n}\right)$ 
for any $\mu$ satisfying (3.8). On the other hand, a direct calculation shows that $\Phi(\infty)=0$ and that

$$
\Phi^{+}(\underline{X})-\Phi^{-}(\underline{X})=g(\underline{X}), \quad \underline{X} \in \Gamma,
$$

where

$$
\Phi^{ \pm}(\underline{X})=\lim _{\Omega^{ \pm} \ni \underline{Y} \rightarrow \underline{X}} \Phi(\underline{Y}) .
$$

Finally, the monogenicity of $\Phi$ is a direct consequence of the well-known fact that the Téodorescu transform constitutes a right inverse of the Dirac operator.

Summarizing, any function $g \in C^{0, v}(\Gamma)$, with $v$ as in (3.7), can be decomposed as

$$
g=g^{+}-g^{-}
$$

where the components $g^{ \pm}$are extendable to monogenic functions in the interior and the exterior of the domain $\Omega$, with respect to $\partial_{\underline{X}}$ and $\partial_{\underline{X}}$, respectively. Note that a decomposition of type (3.12) is said to be of class $C_{0, \mu}$ if $g^{ \pm} \in C^{0, \mu}\left(\overline{\Omega^{ \pm}}\right)$. The remaining question is whether the decomposition (3.12) is unique. In order to investigate this, we will need the following version of the Dolzhenko theorem, as proved in [22].

Theorem 3.3. Let the compact set $\mathbf{E} \subset \mathbb{R}^{2 n}$ be such that $\alpha_{H}(\mathbf{E})<2 n+\mu-\mathbf{1}(0<\mu \leq 1)$. Then, $a$ function $g \in C^{0, \mu}\left(\mathbb{R}^{2 n}\right)$ which is monogenic in $\mathbb{R}^{2 n} \backslash \mathbf{E}$ is monogenic in the whole of $\mathbb{R}^{2 n}$.

We then arrive at the following result.

Theorem 3.4. Suppose that

$$
\alpha_{H}(\Gamma)-2 n+1<\mu<\frac{2 n v-\alpha(\Gamma)}{2 n-\alpha(\Gamma)}
$$

Then, for any $g$ in $C^{0, v}(\Gamma), 0<v \leq 1$, there exists a unique decomposition (3.12) of class $C_{0, \mu}$.

Proof. The existence being shown above, it remains to prove the uniqueness. To this end, assume that $g$ admits two decompositions of class $C_{0, \mu}$, denoted by $f^{ \pm}$and $h^{ \pm}$, respectively. Then

$$
f^{+}-f^{-}=g=h^{+}-h^{-}, \quad \text { on } \Gamma,
$$

implying that

$$
f^{+}-h^{+}=f^{-}-h^{-}, \quad \text { on } \Gamma \text {. }
$$

Consequently, the function $f-h$ is monogenic in $\mathbb{R}^{2 n} \backslash \Gamma$ and belongs to $C^{0, \mu}\left(\mathbb{R}^{2 n}\right)$, whence it is monogenic in $\mathbb{R}^{2 n}$ on account of Theorem 3.3, while it also vanishes at $\infty$. By Liouville's Theorem we conclude that $f \equiv h$. 


\section{A Matricial Hermitean Téodorescu Transform}

A first step in the solution of the Hermitean matrix decomposition problem is the introduction of the matricial Hermitean Téodorescu transform:

$$
\tau_{\Omega} \mathbf{G}_{2}^{1}(\underline{Y})=-\int_{\Omega} \varepsilon(\underline{Z}-\underline{V}) \mathbf{G}_{2}^{1}(\underline{X}) d W\left(\underline{Z}, \underline{Z}^{\dagger}\right)
$$

where $d W\left(\underline{Z}, \underline{Z}^{\dagger}\right)$ is the associated volume element given by

$$
d V(\underline{X})=(-1)^{n(n-1) / 2}\left(\frac{i}{2}\right)^{n} d W\left(\underline{Z}, \underline{Z}^{\dagger}\right)
$$

and $\left(\underline{V}, \underline{V}^{\dagger}\right)$ denote the Hermitean counterparts of $(\underline{Y}, \underline{Y} \mid)$, that is,

$$
\begin{aligned}
\underline{V} & =\frac{1}{2}(\underline{Y}+i \underline{Y} \mid), \\
\underline{V}^{\dagger} & =-\frac{1}{2}(\underline{Y}-i \underline{Y} \mid) .
\end{aligned}
$$

It clearly holds that $\tau_{\Omega} \mathrm{G}_{2}^{1}(\infty)=\mathbf{O}$.

A direct calculation reveals that the Hermitean Téodorescu transform $\tau_{\Omega}$ can be expresed in terms of the Euclidean Téodorescu transforms $\tau_{\Omega}$ and $\tau_{\Omega} \mid$ as follows (see [15]):

$$
\tau_{\Omega} G_{2}^{1}=(-1)^{n(n+1) / 2}(2 i)^{n}\left(\begin{array}{cc}
\tau_{\Omega}\left[g_{1}-g_{2}\right]+i \tau_{\Omega} \mid\left[g_{1}+g_{2}\right] & -\tau_{\Omega}\left[g_{1}-g_{2}\right]+i \tau_{\Omega} \mid\left[g_{1}+g_{2}\right] \\
-\tau_{\Omega}\left[g_{1}-g_{2}\right]+i \tau_{\Omega} \mid\left[g_{1}+g_{2}\right] & \tau_{\Omega}\left[g_{1}-g_{2}\right]+i \tau_{\Omega} \mid\left[g_{1}+g_{2}\right]
\end{array}\right) .
$$

In particular, for the special case of the matrix function $\mathbf{G}_{0}$ (i.e., $g_{1}=g$ and $g_{2}=0$ ) this expression reduces to

$$
\tau_{\Omega} \mathbf{G}_{0}=(-1)^{n(n+1) / 2}(2 i)^{n}\left(\begin{array}{cc}
\tau_{\Omega}[g]+i \tau_{\Omega} \mid[g] & -\tau_{\Omega}[g]+i \tau_{\Omega} \mid[g] \\
-\tau_{\Omega}[g]+i \tau_{\Omega} \mid[g] & \tau_{\Omega}[g]+i \tau_{\Omega} \mid[g]
\end{array}\right)
$$

In what follows we will denote by $\widetilde{G}_{2}^{1}$ the Whitney extension of $\mathbf{G}_{2}^{1}$, that is,

$$
\widetilde{\mathbf{G}}_{2}^{1}=\left(\begin{array}{cc}
\tilde{g}_{1} & \tilde{g}_{2} \\
\tilde{g}_{2} & \tilde{g}_{1}
\end{array}\right)
$$

The following theorem then contains some of its basic properties of the matricial Hermitean Téodorescu transform. They can be proven using standard techniques applied to the present matrix context. 
Theorem 4.1. (i) If $\mathbf{G}_{2}^{1} \in \mathbf{L}_{p}(\Omega)$, with $p>2 n$, then $\boldsymbol{\tau}_{\Omega} \mathbf{G}_{2}^{1} \in \mathcal{C}^{0,(p-2 n) / p}\left(\mathbb{R}^{2 n}\right)$.

(ii) If $\mathbf{G}_{2}^{1} \in \mathbf{L}_{1}(\Omega)$, then

$$
\boldsymbol{\Phi}_{\left(\underline{Z}, \underline{Z}^{\dagger}\right)} \boldsymbol{\tau}_{\Omega} \mathrm{G}_{2}^{1}(\underline{Y})= \begin{cases}(-1)^{n(n+1) / 2}(2 i)^{n} \mathrm{G}_{2}^{1}(\underline{Y}), & \underline{Y} \in \Omega^{+}, \\ 0, & \underline{Y} \in \Omega^{-} .\end{cases}
$$

(iii) If $\mathrm{G}_{2}^{1} \in \mathcal{C}^{0, v}(\Gamma)$ with $v$ as in (3.7), then

$$
\boldsymbol{\tau}_{\Omega} \boldsymbol{\nexists}_{\left(\underline{Z}, \underline{Z}^{\dagger}\right)} \tilde{\mathbf{G}}_{2}^{1} \in \mathcal{C}^{0, \mu}\left(\mathbb{R}^{2 n}\right)
$$

for any $\mu$ satisfying (3.8).

\section{The H-Monogenic Decomposition Problem}

We are now in the possibility to treat the $\mathbf{H}$-monogenic decomposition problem; it means to study under which conditions a given matrix function $\mathrm{G}_{2}^{1} \in \mathcal{C}^{0, v}(\Gamma)$ can be decomposed as

$$
\mathrm{G}_{2}^{1}=\mathrm{G}_{2}^{1^{+}}-\mathrm{G}_{2}^{1^{-}}
$$

where the components $\mathrm{G}_{2}^{1^{ \pm}}$, are Hölder continuous matrix functions on $\Gamma$, which are Hermitean monogenically extendable to $\Omega^{ \pm}$respectively, and moreover $\mathrm{G}_{2}^{1^{-}}(\infty)=\mathrm{O}$. The following theorem provides an answer to that question.

Theorem 5.1. Let $\mathbf{G}_{2}^{1} \in \mathcal{C}^{0, v}(\Gamma)$, with $v$ as in (3.7). Then $\mathbf{G}_{2}^{1}$ admits the Hermitean monogenic decomposition (5.1), where the components are explicitly given by

$$
\begin{aligned}
& \mathbf{G}_{2}^{1^{+}}=\tilde{\mathbf{G}}_{2}^{1}-\frac{(-1)^{n(n+1) / 2}}{(2 i)^{n}} \tau_{\Omega} \boldsymbol{\Xi}_{\left(\underline{Z}, \underline{Z}^{\dagger}\right)} \tilde{\mathbf{G}}_{2}^{1} \\
& \mathbf{G}_{2}^{1^{-}}=\frac{(-1)^{n(n+1) / 2}}{(2 i)^{n}} \tau_{\Omega} \boldsymbol{\Xi}_{\left(\underline{Z}, \underline{Z}^{\dagger}\right)} \tilde{\mathbf{G}}_{2}^{1} .
\end{aligned}
$$

Proof. On account of the assumption on $v$, it follows that $\boldsymbol{\Phi}_{\left(\underline{Z}, \underline{Z}^{\dagger}\right)} \tilde{\mathbf{G}}_{2}^{1}$ belongs to $\mathbf{L}_{p}(\Omega)$, for $p=1$ and $p>2 n$, simultaneously. Then, the Hölder continuity of $\mathbf{G}_{2}^{1^{ \pm}}$directly follows from Theorem 4.1, (i) and (iii). Next, the matrix inversion formula (ii) in Theorem 4.1 yields

$$
\boldsymbol{\Phi}_{\left(\underline{Z}, \underline{\zeta}^{\dagger}\right)} \mathbf{G}_{2}^{1^{ \pm}}=\mathbf{O} \quad \text { in } \mathbb{R}^{2 n} \backslash \Gamma
$$

showing the $\mathbf{H}$-monogenicity of $\mathrm{G}_{2}^{1^{ \pm}}$in $\Omega^{ \pm}$, respectively. 
In order to rephrase Theorem 5.1 in the $h$-monogenic setting, as studied in [23], we only need to ensure that

$$
\begin{aligned}
& \frac{(-1)^{n(n+1) / 2}}{(2 i)^{n}} \tau_{\Omega} \boldsymbol{\Phi}_{\left(\underline{Z}, \underline{Z}^{\dagger}\right)} \tilde{\mathbf{G}}_{0} \\
& \quad=\left(\begin{array}{cc}
\tau_{\Omega}\left[\partial_{\underline{Z}} \tilde{g}-\partial_{\underline{Z}^{\dagger}} \tilde{g}\right]+i \tau_{\Omega} \mid\left[\partial_{\underline{Z}} \underline{\tilde{g}}+\partial_{\underline{Z^{\dagger}} \tilde{g}}\right] & 0 \\
0 & \tau_{\Omega}\left[\partial_{\underline{Z}} \tilde{g}-\partial_{\underline{Z}^{+}}\right]+i \tau_{\Omega} \mid\left[\partial_{\underline{Z}} \tilde{g}+\partial_{\underline{Z}^{+}} \tilde{g}\right]
\end{array}\right)
\end{aligned}
$$

or, equivalently, that

$$
\tau_{\Omega}\left[\partial_{\underline{Z}} \tilde{g}-\partial_{\underline{Z}^{\dagger}} \tilde{g}\right]=i \tau_{\Omega} \mid\left[\partial_{\underline{Z}} \tilde{g}+\partial_{\underline{Z}^{\dagger}} \tilde{g}\right]
$$

which, by means of some direct calculations, can be rewritten as

$$
\tau_{\Omega} \partial_{\underline{X}} \tilde{g}=\tau_{\Omega}\left|\partial_{\underline{X}}\right| \tilde{g}
$$

This is precisely the condition under which a function $g \in C^{0, v}(\Gamma)$ has been found to admit an $h$-monogenic decomposition (3.12); see [23, Theorem 2]. This result may be reformulated into the present setting as follows.

Theorem 5.2. Let $g \in C^{0, v}(\Gamma)$, with $v$ as in (3.7), and consider the corresponding matrix function $\mathbf{G}_{0} \in \mathcal{C}^{0, v}(\Gamma)$. Then $\mathbf{G}_{0}$ admits the decomposition (5.1) in terms of h-monogenic functions if and only if (5.4) holds.

Remark 5.3. Even though the decomposition (5.1) is not unique in general, it will be so in the corresponding class:

$$
\mathcal{C}_{0, \mu}=\left\{\mathbf{G}_{2}^{1} \mid g_{1}, g_{2} \in C_{0, \mu}\right\}
$$

for $\alpha_{H}(\Gamma)-2 n+1<\mu<(2 n v-\alpha(\Gamma)) /(2 n-\alpha(\Gamma))$; see Theorem 3.4.

Remark 5.4. When $\Gamma$ is sufficiently regular, for example, Ahlfors-David regular, the expressions (5.2) reduce to the ones obtained in [17] in terms of the matricial Hermitean Cauchy integral, the latter being easily obtained using the Hermitean Borel-Pompeiu formula, as proved in [16]. Indeed, applying this Borel-Pompeiu formula to $\widetilde{\mathrm{G}}_{2}^{1}$, we obtain

$$
\mathcal{C}_{\Gamma} \tilde{\mathbf{G}}_{2}^{1}(\underline{Y})+\tau_{\Omega} \boldsymbol{\Phi}_{\left(\underline{Z}, \underline{Z}^{\dagger}\right)} \tilde{\mathbf{G}}_{2}^{1}(\underline{Y})= \begin{cases}(-1)^{n(n+1) / 2}(2 i)^{n} \widetilde{\mathbf{G}}_{2}^{1}(\underline{Y}), & \underline{Y} \in \Omega^{+}, \\ 0, & \underline{Y} \in \Omega^{-},\end{cases}
$$


where $\mathcal{C}_{\Gamma} \tilde{\mathbf{G}}_{2}^{1}(\underline{Y})$ is the Hermitean Cauchy integral given by

$$
\mathcal{C}_{\Gamma} \tilde{\mathbf{G}}_{2}^{1}(\underline{Y})=\int_{\Gamma} \mathcal{E}(\underline{Z}-\underline{V}) \mathbf{N}_{\left(\underline{Z}, \underline{Z}^{\dagger}\right)} \tilde{\mathbf{G}}_{2}^{1}(\underline{X}) d \mathscr{\ell}^{2 n-1}, \quad \underline{Y} \in \Omega^{ \pm}
$$

Here, the additional circulant matrix

$$
\mathbf{N}_{\left(\underline{Z}, \underline{Z}^{\dagger}\right)}=\left(\begin{array}{cc}
\underline{N} & -\underline{N^{\dagger}} \\
-\underline{N}^{\dagger} & \underline{N}
\end{array}\right)
$$

contains (up to a factor) the Hermitean projections $\underline{N}$ and $\underline{N}^{\dagger}$ of the outward unit normal vector $\underline{n}(\underline{X})$ at the point $\underline{X}$, while the matrix Hausdorff measure $\mathrm{d} \mathscr{\ell}^{2 n-1}$ is given by

$$
\mathrm{d} \mathscr{\ell}^{2 n-1}=\left(\begin{array}{cc}
d \mathscr{H}^{2 n-1} & 0 \\
0 & d \mathscr{\ell}^{2 n-1}
\end{array}\right) .
$$

Since $\left.\tilde{\mathbf{G}}_{2}^{1}\right|_{\Gamma}=\mathbf{G}_{2}^{1}$, we have

$$
\mathcal{C}_{\Gamma} \mathbf{G}_{2}^{1}(\underline{Y})+\boldsymbol{\tau}_{\Omega} \boldsymbol{\Phi}_{\left(\underline{Z}, \underline{Z}^{\dagger}\right)} \tilde{\mathbf{G}}_{2}^{1}(\underline{Y}) \cdot= \begin{cases}(-1)^{n(n+1) / 2}(2 i)^{n} \tilde{\mathbf{G}}_{2}^{1}(\underline{Y}), & \underline{Y} \in \Omega^{+}, \\ 0, & \underline{Y} \in \Omega^{-}\end{cases}
$$

or, using the notations of (5.2),

$$
\frac{1}{(-1)^{n(n+1) / 2}(2 i)^{n}} \mathcal{C}_{\Gamma} \mathrm{G}_{2}^{1}(\underline{Y})= \begin{cases}\mathrm{G}_{2}^{+}(\underline{Y}), & \underline{Y} \in \Omega^{+}, \\ \mathrm{G}_{2}^{1^{-}}(\underline{Y}), & \underline{Y} \in \Omega^{-},\end{cases}
$$

which coincides with [17, equation (5.2)].

\section{Acknowledgments}

This paper was written during a scientific stay of the first author at the Clifford Research Group of the Department of Mathematical Analysis of Ghent University, supported by a "Visiting Postdoctoral Fellowship" of the Flemish Research Foundation. He wishes to thank the members of the Clifford Research Group for their kind hospitality during this stay.

\section{References}

[1] F. Brackx, R. Delanghe, and F. Sommen, Clifford Analysis, vol. 76 of Research Notes in Mathematics, Pitman, Boston, Mass, USA, 1982.

[2] R. Delanghe, F. Sommen, and V. Souček, Clifford Algebra and Spinor-Valued Functions, vol. 53, Kluwer Academic Publishers, Dordrecht, The Netherlands, 1992.

[3] K. Gürlebeck and W. Sprössig, Quaternionic and Clifford Calculus for Physicists and Engineers, John Wiley \& Sons, Chichester, UK, 1998. 
[4] J. Gilbert and M. Murray, Clifford Algebras and Dirac Operators in Harmonic Analysis, vol. 26, Cambridge University Press, Cambridge, UK, 1991.

[5] K. Gürlebeck, K. Habetha, and W. Sprössig, Holomorphic Functions in the Plane and n-Dimensional Space, Birkhäuser, Basel, Switzerland, 2008, translated from the 2006 German original.

[6] F. Brackx, H. De Schepper, and F. Sommen, "The Hermitean Clifford analysis toolbox," Advances in Applied Clifford Algebras, vol. 18, no. 3-4, pp. 451-487, 2008.

[7] J. Ryan, "Complexified Clifford analysis," Complex Variables. Theory and Application, vol. 1, no. 1, pp. 119-149, 1982-1983.

[8] R. Rocha-Chávez, M. Shapiro, and F. Sommen, Integral Theorems for Functions and Differential Forms in $\mathbb{C}^{m}$, vol. 428 of Research Notes in Mathematics, Chapman \& Hall/CRC, Boca Raton, Fla, USA, 2002.

[9] I. Sabadini and F. Sommen, "Hermitian Clifford analysis and resolutions," Mathematical Methods in the Applied Sciences, vol. 25, no. 16-18, pp. 1395-1413, 2002.

[10] F. Colombo, I. Sabadini, F. Sommen, and D. C. Struppa, Analysis of Dirac Systems and Computational Algebra, vol. 39, Birkhäuser, Boston, Mass, USA, 2004.

[11] F. Brackx, J. Bureš, H. De Schepper, D. Eelbode, F. Sommen, and V. Souček, "Fundaments of Hermitean Clifford analysis-part I: complex structure," Complex Analysis and Operator Theory, vol. 1, no. 3, pp. 341-365, 2007.

[12] F. Brackx, J. Bureš, H. De Schepper, D. Eelbode, F. Sommen, and V. Souček, "Fundaments of Hermitean Clifford analysis-partII: splitting of $h$-monogenic equations," Complex Variables and Elliptic Equations, vol. 52, no. 10-11, pp. 1063-1079, 2007.

[13] F. Brackx, H. De Schepper, and F. Sommen, "A theoretical framework for wavelet analysis in a Hermitean Clifford setting," Communications on Pure and Applied Analysis, vol. 6, no. 3, pp. 549-567, 2007.

[14] D. Eelbode, "Stirling numbers and spin-Euler polynomials," Experimental Mathematics, vol. 16, no. 1, pp. 55-66, 2007.

[15] F. Brackx, H. De Schepper, M. E. Luna Elizarrars, and M. Shapiro, "Integral representation formulae in Hermitean Clifford analysis," in Proceedings of the 18th International Conference on the Application of Computer Science and Mathematics in Architecture and Civil Engineering, K. Gürlebeck and C. Könke, Eds., Weimar, Germany, July 2009.

[16] F. Brackx, B. De Knock, H. De Schepper, and F. Sommen, “On Cauchy and Martinelli-Bochner integral formulae in Hermitean Clifford analysis," Bulletin of the Brazilian Mathematical Society, vol. 40, no. 3, pp. 395-416, 2009.

[17] R. Abreu-Blaya, J. Bory-Reyes, F. Brackx, et al., "Hermitean Cauchy integral decomposition of continuous functions on hypersurfaces," Boundary Value Problems, vol. 2008, Article ID 425256, 16 pages, 2008.

[18] F. Brackx, B. De Knock, and H. De Schepper, "A matrix Hilbert transform in Hermitean Clifford analysis," Journal of Mathematical Analysis and Applications, vol. 344, no. 2, pp. 1068-1078, 2008.

[19] R. Abreu-Blaya and J. Bory-Reyes, "A Martinelli-Bochner formula on fractal domains," Archiv der Mathematik, vol. 92, no. 4, pp. 335-343, 2009.

[20] R. Abreu-Blaya, J. Bory-Reyes, and T. Moreno García, “Minkowski dimension and Cauchy transform in Clifford analysis," Complex Analysis and Operator Theory, vol. 1, no. 3, pp. 301-315, 2007.

[21] R. Abreu-Blaya, J. Bory-Reyes, and T. Moreno-García, "Cauchy transform on nonrectifiable surfaces in Clifford analysis," Journal of Mathematical Analysis and Applications, vol. 339, no. 1, pp. 31-44, 2008.

[22] R. Abreu-Blaya, J. Bory-Reyes, and D. Peña-Peña, "Jump problem and removable singularities for monogenic functions," The Journal of Geometric Analysis, vol. 17, no. 1, pp. 1-13, 2007.

[23] R. Abreu-Blaya, J. Bory-Reyes, and T. Moreno García, "Hermitian decomposition of continuous functions on a fractal surface," Bulletin of the Brazilian Mathematical Society, vol. 40, no. 1, pp. 107$115,2009$.

[24] R. Abreu-Blaya, J. Bory-Reyes, D. Peña Peña, and F. Sommen, “A boundary value problem for Hermitian monogenic functions," Boundary Value Problems, vol. 2008, 7 pages, 2008.

[25] K. J. Falconer, The Geometry of Fractal Sets, vol. 85 of Cambridge Tracts in Mathematics, Cambridge University Press, Cambridge, UK, 1986.

[26] J. Feder, Fractals, Physics of Solids and Liquids, Plenum Press, New York, NY, USA, 1988, with a foreword by Benoit B. Mandelbro.

[27] M. L. Lapidus and H. Maier, "Hypothèse de Riemann, cordes fractales vibrantes et conjecture de Weyl-Berry modifiée," Comptes Rendus de l'Académie des Sciences. Série I. Mathématique, vol. 313, no. 1, pp. 19-24, 1991 (French). 
[28] H. Federer, Geometric Measure Theory, Die Grundlehren der mathematischen Wissenschaften 153, Springer, New York, NY, USA, 1969.

[29] Elias M. Stein, Singular Integrals and Differentiability Properties of Functions, Princeton Mathematical Series, no. 30, Princeton University Press, Princeton, NJ, USA, 1970.

[30] J. Harrison and A. Norton, "The Gauss-Green theorem for fractal boundaries," Duke Mathematical Journal, vol. 67 , no. 3, pp. 575-588, 1992. 